\title{
WiP Abstract: Platform for Security-Aware Design of Human-on-the-Loop Cyber-Physical Systems
}

\author{
Mahmoud Elfar Haibei Zhu Adithya Raghunathan Yi Y. Tay Jeffrey Wubbenhorst \\ M. L. Cummings Miroslav Pajic \\ Duke University \\ \{mahmoud.elfar, haibei.zhu, ra102, yt61, jsw51\}@duke.edu \\ \{mary.cummings, miroslav.pajic\}@duke.edu
}

\begin{abstract}
Cyber-Physical Systems (CPS) are commonly supervisory control systems where a human-on-the-loop (HOL) supervises one or more autonomous systems, while embedded autonomy allows the operators to intermittently attend to the system and other tasks. Thus, it is imperative that the design of any security-aware CPS considers the impact of the human interaction with the system on security guarantees. Yet, there has been very little work on design of Human-CPS that promotes human situational awareness for enhanced system performance, particularly in terms of cyber-physical security and real-time defense against attacks. One of the main obstacles to the rapid advancement of this field is the scarcity of testbeds for evaluating security-aware Human-CPS interactions.

We present RESCHU-SA, an extendable virtual platform that facilitates studying the impact that HOL has on security of CPS with varying levels of autonomy. It allows users to analyze how inductive reasoning and ability to provide context, particularly during an attack, affects the overall CPS security guarantees. The proposed platform is an extension of the Research Environment for Supervisory Control of Heterogeneous Unmanned Vehicles (RESCHU) simulation environment, previously used in various applications including studies focused on supervising Unmanned Aerial Vehicles (UAVs) missions and evaluation of interface usability.
\end{abstract}

\section{CCS CONCEPTS}

-Human-centered computing $\rightarrow$ Laboratory experiments; Systems and tools for interaction design; $\bullet$ Security and privacy $\rightarrow$ Domainspecific security and privacy architectures;

\section{KEYWORDS}

CPS Security, Human-on-the-Loop CPS, Unmanned Aerial Vehicles

\section{ACM Reference format:}

Mahmoud Elfar Haibei Zhu Adithya Raghunathan YiY. Tay Jeffrey Wubbenhorst and M. L. Cummings Miroslav Pajic. 2017. WiP Abstract: Platform for Security-Aware Design of Human-on-the-Loop CyberPhysical Systems. In Proceedings of The 8th ACM/IEEE International Conference on Cyber-Physical Systems, Pittsburgh, PA USA, April 2017 (ICCPS), 1 pages.

DOI: $10.1145 / 3055004.3064846$

This work was supported in part by the NSF CNS-1505701 grant and the Intel-NSF Partnership for Cyber-Physical Systems Security and Privacy. This material is also based on research sponsored by the ONR under agreement number N00014-17-1-2012. Permission to make digital or hard copies of part or all of this work for personal or classroom use is granted without fee provided that copies are not made or distributed for profit or commercial advantage and that copies bear this notice and the full citation on the first page. Copyrights for third-party components of this work must be honored. For all other uses, contact the owner/author(s).

ICCPS, Pittsburgh, PA USA

(c) 2017 Copyright held by the owner/author(s). 978-1-4503-4965-9/17/04 ..\$15.00 DOI: $10.1145 / 3055004.3064846$

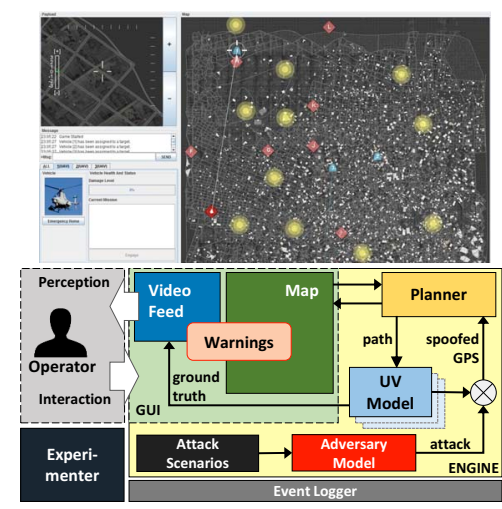

Figure 1: RESCHU-SA interface (top) and architecture.

\section{DEMONSTRATION}

In our demo, we illustrate the developed platform on multi-UAV missions supervised by a single operator, where some of the UAVs may be compromised. The RESCHU-SA operator interface consists of a map area capturing information about UAVs (e.g., their flight plans, targets, threat zones and expected time of arrival), a camerafeed display from the selected UAV, and a communication panel (Fig. 1). Through the graphical user interface (GUI), the operator can supervise a fleet of UAVs on a timed mission. Each mission requires the UAVs to visit several target locations, at which the operator should complete a set of visual tasks using the camera feed.

According to predefined attack scenarios, some UAVs are attacked by injecting malicious GPS data into the planner of the corresponding UAVs and hence affecting information reported to the user. Due to the stealthy nature of such attacks, the reported location of a compromised UAV slowly changes over time, limiting the operator's ability to detect such attacks from the displayed UAV's trajectory on the map. However, as a UAV live video feed always reflects the ground truth, the operator may detect an attack if she can identify discrepancies between the video feed and the reported location, allowing analysis of the operator's situational awareness.

Due to the modular platform design, the system designer can extend both the attack models and UAV dynamics. The current attack model allows for configuring adversarial behavior, including aggressive vs. stealthy attacks, timing, target regions, and warnings raised by intrusion detection systems. We also use randomized maps containing regions with various feature richness to enable studying how feature density influences the operator's ability to identify an UAV's location from the video feed. Unlike conventional camera feeds that show sporadic static images of predefined areas on the map, RESCHU-SA emulates continuous video feeds and supports a number of features that can increase video fidelity, including noise overlay, vehicle vibrations, and communication delays. 